\title{
The Adjustable Capacity Transformer Economical Analysis Based on Medium and Long-term Load Forecasting
}

\author{
WANG Qian ${ }^{1, a}$, MENG Lu ${ }^{2, b}$, Niu Yu ${ }^{3, c}$,Yang Xinyong ${ }^{3, d}$ \\ ${ }^{1}$ HAEPC Electric Power Research Institute, Zhengzhou, Henan, China \\ ${ }^{2}$ Zhengzhou Power Supply Company, Zhengzhou, Henan, China \\ ${ }^{3}$ Henan EnPai hi-tech group co., LTD, Zhengzhou, Henan, China \\ a wangqian0523@yahoo.com.cn, ${ }^{\mathrm{b}}$ menghd2009@163.com
}

\begin{abstract}
Keywords: medium and long-term load forecasting; adjustable capacity transformer; economic analysis model of the transformer; Loss cost model

Abstract. In recent years, the residential electricity load characteristic changes significantly. Especially in rural areas, the load is low at ordinary time, but the peak load rise sharply in Spring Festival. In response to this situation and to improve distribution transformer economical operation, adjustable capacity transformer was developed and applied. But the suitable load characteristics of the adjustable capacity transformer is qualitative given, also did not consider the growth of the load within adjustable capacity transformer life. If choose undeserved, it not only cause the waste of investment, even may cause loss of increase. The development of medium and long-term load forecasting applicability research and evaluation of energy conservation of adjustable capacity transformer is important significance to guide energy conservation and accurate distribution network investment.
\end{abstract}

\section{Introduction}

In recent years, the change of rural life style changes the load features obviously, for example, Load decentralization, strong seasonality, lower average load, big peak valley difference, rapid load growth in the Spring Festival and farming season. The load characteristic leads to the rural distribution transformer very low load rate at ordinary times, capacity waste, and larger loss compared with small capacity. High overload would happen in high load period, and it is a threat to the equipment stable operation and reliable power supply.

Adjustable capacity transformer is produced for rural power grids which have obvious seasonal difference between peak and valley electricity load. Adjustable capacity transformer has two rated capacity, ratio of about $3: 1$, and the two rated capacity can be seamlessly switch automatically. Small capacity is used in small load, large capacity is used in heavy load, so that we can reduce the wastage of the distribution transformer. But the load suitable for the adjustable capacity transformer is given qualitatively, not quantitatively. If the capacity of the switch point set is not accurate, the loss not only does not be reduce, may also be increased. In addition, when the transformer is chosen , long-term load growth should be considered. The cost of adjustable capacity transformer is about 1.5 times that of the ordinary transformer. So if choose is undeserved, the investment is enlarged, and the loss is increased.

In this paper, the principle of the adjustable capacity transformer will be researched, the application scope of adjustable capacity transformer will be researched quantitatively, and medium and long-term load forecasting model will be studied. Based on the analysis of medium and long-term load forecasting, we analysis the economy of life range of adjustable capacity transformer and provide guidance for the selection of capacity transformer.

All manuscripts must be in English, also the table and figure texts, otherwise we cannot publish your paper. 


\section{Adjustable Capacity Transformer}

Adjustable capacity transformer has two rated capacity, and the ratio of the two capacity is about $3: 1$. Under the condition of the output voltage does not change, it can realize the capacity of two kinds of switches, small load with small capacity, load large with large capacity. Adjustable capacity transformer is suitable for load dispersion, large difference of seasonal power consumption and low average load rate. Adjustable capacity transformer in practical operation, according to the load conditions, the high voltage winding implements $\Delta$ connection and Y connection, and the low voltage winding realize "parallel" and "series" transformation, so as to realize the transformation of two kinds of nominal capacity. When the big rated capacity adjusted to small rated capacity, the output voltage remains the same. Because the input voltage under small rated capacity state is $1 / \sqrt{3}$ of that under big rated capacity state, and output winding process number of turns under small rated capacity state is $\sqrt{3}$ times of that under big rated capacity state. At the same time, due to the increase of the low voltage winding circle number, no-load loss and no-load current is reduced, to achieve the goal of the loss reduction and energy saving.

\section{Adjustable capacity transformer loss analysis}

The total loss of transformer is equal to no-load loss and load loss. No-load loss is the iron loss of transformer, which is a constant. The short circuit loss is refers to the loss under rated operation, which is the loss under full load. Load loss is proportional to the square of the current, load factor is equal to the ratio of the actual current and rated current, and load loss is the square of load factor timing the short circuit losses.

$$
\begin{gathered}
\mathrm{P}_{\text {total }}=\mathrm{P}_{0}+\mathrm{P}_{\text {load }} \\
\mathrm{P}_{0}=\mathrm{P}_{\mathrm{Fe}} \\
\beta=\frac{\mathrm{P}_{\text {load }}}{\mathrm{P}_{\text {rated }}}=\frac{\mathrm{I}_{\text {load }} \times \mathrm{U}}{\mathrm{I}_{\text {rated }} \times \mathrm{U}}=\frac{\mathrm{I}_{\text {load }}}{\mathrm{I}_{\text {rated }}} \\
\mathrm{P}_{\text {load }}=\mathrm{I}_{\text {load }}^{2} \times \mathrm{R}=\beta^{2} \cdot \mathrm{I}_{\text {rated }}^{2} \cdot \mathrm{R}=\beta^{2} \cdot \mathrm{P}_{\mathrm{k}}
\end{gathered}
$$

Among them, ${ }^{P_{0}}$ is the no-load loss of transformer, and $\beta$ is the transformer load rate.

For adjustable capacity transformer, we change size gear according to the load condition, by changing the transformer rated capacity to achieve the purpose of energy saving. No-load loss and load loss of capacity transformer is opposite bigger under large capacity cuts, no-load loss and load loss of capacity transformer is relatively small under small capacity cuts. Under two kinds of capacity loss, the calculation formula is same, and the parameters is just slightly different.

$$
\begin{aligned}
& \mathrm{P}_{\text {total }}=\mathrm{P}_{H}+\mathrm{P}_{L} \\
& \quad=\mathrm{P}_{0 H}+\mathrm{P}_{\text {load. }}+\mathrm{P}_{0 L}+\mathrm{P}_{1 \text { oad. } \mathrm{L}} \\
& \quad=\mathrm{P}_{0 H}+\beta_{H}{ }^{2} \cdot \mathrm{P}_{\mathrm{kH}}+\mathrm{P}_{0 \mathrm{~L}}+\beta_{\mathrm{L}}{ }^{2} \cdot \mathrm{P}_{\mathrm{kL}}
\end{aligned}
$$

Among them, $\mathrm{P}_{H}$ is the no-load loss of adjustable capacity transformer, ${ }_{\mathrm{L}}$ is the short-circuit loss of adjustable capacity transformer, $\mathrm{P}_{0 \mathrm{H}}$ is the no-load loss of adjustable capacity transformer under large capacity, $\mathrm{P}_{0 \mathrm{~L}}$ is the no-load loss of adjustable capacity transformer under small capacity, $\mathrm{P}_{k \mathrm{H}}$ is the short-circuit loss of adjustable capacity transformer under large capacity, $\mathrm{P}_{k \mathrm{~L}}$ is the short-circuit loss of adjustable capacity transformer under small capacity, $\beta_{H}$ is the load rate of adjustable capacity transformer under large capacity, $\beta_{\mathrm{L}}$ is the load rate of adjustable capacity transformer under small capacity. 


\section{Adjustable capacity transformer loss reduction analysis}

Adjustable capacity transformer has two capacity gear, so loss curve is determined according to the capacity of the switch point of two parts. Under different capacity switch point, adjustable capacity transformer loss is different under the same load cases. The optimal switch point is the point when the loss is the same no matter under small capacity model or large capacity mode. Below with s11 adjustable capacity transformer and normal transformer as an example, we analysis the amount of energy saving of adjustable capacity transformer and normal transformer.

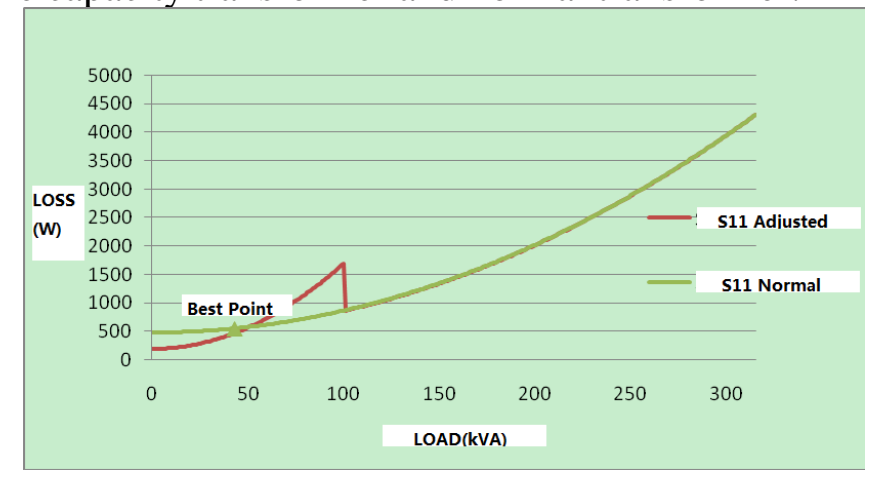

Fig. 1 S11 adjustable capacity transformer and S11 transformer load - ordinary loss

From the above it can be seen that, when the load in the interval of [100,315], the loss curve of adjustable capacity transformer and ordinary transformer is the same. When the load in the interval of [0, the optimal switching point], the loss of adjustable capacity transformer is lower than ordinary transformer. When the load in the interval of [the best switching capacity, 100], the loss of adjustable capacity transformer is higher than ordinary transformer.

\section{Medium and long-term load forecasting}

In recent years, the on-line monitoring equipment of distribution transformer is widely used, and variable load test data accumulated gradually, which provides a large number of basic data for load forecast. Medium and long-term load forecasting is changeable for the next few years to decades of load forecasting. Accurate to predict medium and long-term load forecasting provides the basis for the construction of the grid size, construction distribution, energy balance, the balance of power capital and human resources.

\section{The characteristics of the medium and long-term load forecasting}

Compared with short-term load forecasting, medium and long term load forecasting of distribution network has the following characteristics.(1)Non-stationary variation. Medium and long-term load forecasting is the dual influence of deterministic and random changes.(2)Limited historical data, medium and long-term load forecasting needs to analyze the history data and the changing rule of the medium and long term. But we didn't attach importance to distribution network previously, and automatic data collection device is missing, which leading to lack of historical data.(3) Big structure of load change. Time span of medium and long term load forecasting is large. Within the scope of the study time, the composition of the user and power consumption habits change largely, so we can't describe load change trend in a single rule.(4) More impact factor. Distribution network load is influenced by the outside world, such as weather climate, the user habits, and distributed energy etc, in addition to the load characteristics of itself. The relationship among these influence factors is hard to grasp.

\section{Medium and long-term load forecasting algorithm}

Grey prediction model, clustering analysis and fuzzy comprehensive evaluation method are the commonly used model for medium and long-term load forecasting. Due to the characteristics of the 
medium and long-term load forecasting, the fusion algorithms of combination forecast model can obtain higher precision.

(1) Grey forecasting model

Grey forecasting uses the changing process of the system behavior characteristics as a Grey system, using the theory of Grey differential equation Model (Grey Model). If use the model for prediction of a single index of power system, that is to solve the differential equation. The homographic solution time corresponding function expression is the grey forecasting model. Next we use the statistics between fitting value and real value fitting between value and real value of statistics, according to this model to predict the future of the load.

(2) Particle swarm optimization model

Particle swarm optimization algorithm is a new swarm intelligence algorithm after ant colony algorithm, through cooperation and competition between individuals achieve global searching. The principle of particle swarm optimization algorithm with genetic algorithm is similar, system search the optimal value by a group of initial random iterative solution. But unlike genetic algorithm, which by the operation of selecting, crossover and mutation to the individual, particles search in the solution space by following the optimal particle. Its advantage is easy to operate, easy to implement, concise algorithm parameters, and easy to adjust. At present, the PSO of evolutionary computation has become the international research hot spot. So, just a few short years time, PSO algorithm has made rapid progress. And in the fuzzy system control, neural network training, traffic incident detection, power system optimization, optimization of multivariate function, and many other fields has been widely attention and application.

(3) Expert system for medium and long-term prediction method

Expert system prediction method analyze the related influencing factors of load in store of the past years, to collect the knowledge of experienced load forecasting person, and to extract rules according to certain rules of load forecasting. Accurate load forecasting needs not only the support of new and high technology, but also the human experience and wisdom. Therefore, expert system for such technology will be needed. Expert system method is a kind of good method to convert human experience which is not quantitative.

(4) Combination forecast model

In the current, domestic and international research electric power system load forecasting deeply. But the medium and long-term load forecasting is quite tedious power engineering, and influenced by the national economy, population, industrial production index of many factors, such as direct or indirect influence. Therefore, so far, there has been no a prediction model which can ensure that can achieve higher prediction precision in all cases. The favorable information on a single load forecasting model should be used fully, to create a power load combination forecasting model as a more feasible method.

\section{Economic Analysis of adjustable capacity transformer considering load forecasting}

Transformer economical analysis model is a comprehensive model, considering the load characteristics, load growth and volatility within the scope of the transformer life, the transformer purchase cost, operation maintenance cost and wastage. Transformer economical analysis can be used to select of the transformer, to improve the precision of investment, to maximize interests. Especially, the cost of adjustable capacity transformer is about 1.5 times of the ordinary transformer, we must comprehensive consider of the relationship between transformer investment and saving the loss.

\section{Economic efficiency analysis model}

Because this article is the study on medium and long-term load forecasting based transformer economical efficiency, so we use the whole life model.

$$
L C C=\mathrm{C}_{\mathrm{iv}}+\mathrm{C}_{\mathrm{oc}}+\mathrm{C}_{\mathrm{mc}}+C_{\mathrm{dc}}
$$


Among them, ${ }^{\mathrm{C}_{\mathrm{iv}}}$ refers to the acquisition cost of the transformer, including the purchase cost of transformer and its maintenance equipment, staff training expenses and cost of data records; ${ }^{\mathrm{C}_{\mathrm{oc}}}$ refers to the operating costs of transformer, including test, installing, loss, service cost of transformer, labor cost, etc.; ${ }^{C_{m c}}$ refers to the maintenance costs of the transformer, including maintenance costs before fault and repair cost after failure; $C_{\mathrm{dc}}$ refers to the disposal cost of transformer, including the transformer scrap cost and salvage value.

$$
\mathrm{C}_{\mathrm{iv}}=\mathrm{C}_{\mathrm{tp}}+\mathrm{C}_{\mathrm{ep}}+\mathrm{C}_{\mathrm{td}}
$$

Among them, ${ }^{C_{\text {tp }}}$ refers to acquisition cost of transformer; ${ }^{C_{e p}}$ refers to the acquisition cost of transformer; ${ }_{\text {td }}$ refers to the purchase of transformer equipment maintenance cost.

$$
\mathrm{C}_{\mathrm{oc}}=\mathrm{C}_{\mathrm{exc}}+\mathrm{C}_{\mathrm{enc}}+\mathrm{C}_{\mathrm{inc}}+\mathrm{C}_{\mathrm{fc}}+\mathrm{C}_{\mathrm{moc}}
$$

Among them, ${ }^{C_{\text {exc }}}$ refers to the transformer test fee. Transformer should be done some necessary test before put into operation, to complete the testing by equipment technical standards, such as short circuit withstand test, temperature rise test and partial discharge test, etc. ${ }^{\mathrm{C}_{\text {enc }}}$ refers to the loss cost of transformer running, because transformer keeping in the consumption of electrical energy in running state; ${ }^{C_{i n c}}$ refers to the cost of installation of the transformer, including the total cost of transportation, artificial and debugging; $\mathrm{C}_{\mathrm{fc}}$ refers to the shutdown loss cost of the transformer, within the prescribed life cycle, because of the loss caused by failure or efficiency shutdown; $\mathrm{C}_{\text {moc }}$ refers to the labor and other costs in the life of the transformer cycle, responsible for the operation or management personnel salary, as well as related to all expenses of transformer operation and others.

$$
\mathrm{C}_{\mathrm{oc}}=\mathrm{C}_{\mathrm{bf}}-\mathrm{C}_{\mathrm{cz}}
$$

Among them, ${ }^{C_{o c}}$ refers to the disposal cost, ${ }^{C_{b f}}$ refers to the scrap cost, ${ }^{{ }^{c z}}$ refers to the salvage value of the transformer.

The adjustable capacity transformer loss cost model based on the medium and long-term load forecasting

$$
\mathrm{C}_{\mathrm{enc}}=\mathrm{E} \times \sum_{i=1}^{i=k}\left[\left(\mathrm{P}_{\mathrm{OH}} \mathrm{T}_{\mathrm{H} i}+\mathrm{P}_{\mathrm{OL}} \mathrm{T}_{\mathrm{L} i}\right)+\beta_{\mathrm{H} i}^{2} \mathrm{P}_{k \mathrm{H}} \mathrm{T}_{\mathrm{H} i}+\beta_{\mathrm{L} i}^{2} \mathrm{P}_{\mathrm{kL}} \mathrm{T}_{\mathrm{L} i}\right]
$$

Among them, $\mathrm{E}$ is the average sales price for the transformer users, $i$ is the $i$ th years in the transformer life,$k$ is the transformer life limit, ${ }_{\mathrm{H} i}$ is the running time of transformer under big rated capacity, $\mathrm{T}_{\mathrm{L} i}$ is the running time of transformer under small rated capacity, $\beta_{\mathrm{H} i}$ is the average load rate of the transformer under big rated capacity, $\beta_{\mathrm{L} i}$ is the average load rate of the transformer under small rated capacity.

\section{Conclusion}

we analysis the life-cycle cost of adjustable capacity transformer compared with common transformer, based on medium and long-term load forecasting. The research plays a strong guiding role wile selecting a match variable, making the selection more reasonable, more economical, and maximize investment utilization. The next step work is to establish medium and long-term load forecasting model, and to validate the model using an example..

\section{References}

[1] Jun Hk, Kim J H. Life Cycle Cost Modeling for Railway Vehicle[J].1EEE,2007. 
[2] Jiang Yimin. Whole life cycle cost analysis of transformer[J]. Shanghai Electric Powe,2004(3):188-191.

[3] Fang Lihua, Li Jingping, Liu Jianchu. The efficiency of new type energy saving distribution transformer analysis and selection [J]. Southern Power Grid Technology,2009(3):104-106.

[4] Wang Jinli. On-load capacity transformer comprehensive economic analysis and application research [J]. High Voltage Electric Equipment,2009(6):32-35.

[5] Cui Xinqi, Yin Laibin, Fan Chunju. Reform of the substation transformer full life cycle cost (LCC) model of the research [J]. Power system protection and control,2010(4):69-73. 